\title{
cAMP response element binding protein $\mathrm{H}$ mediates fenofibrate-induced suppression of hepatic lipogenesis
}

\author{
A.-K. Min • J. Y. Jeong • Y. Go • Y.-K. Choi • Y.-D. Kim • \\ I.-K. Lee • K.-G. Park
}

Received: 5 June 2012 / Accepted: 18 October 2012 / Published online: 13 November 2012

(C) Springer-Verlag Berlin Heidelberg 2012

\begin{abstract}
Aims/hypothesis Fenofibrate is a drug used to treat hyperlipidaemia that works by inhibiting hepatic triacylglycerol synthesis. Sterol regulatory element binding protein-1c (SREBP-1c) is a major regulator of the expression of genes involved in hepatic triacylglycerol synthesis. In addition, endoplasmic reticulum (ER)-bound transcription factor families are involved in the control of various metabolic pathways. Here, we show a novel function for an ER-bound transcription factor, cAMP response element binding protein $\mathrm{H}$ (CREBH), in fenofibrate-mediated inhibition of hepatic lipogenesis.

Methods The effects of fenofibrate and adenovirus-mediated Crebh (also known as Creb313) overexpression (Ad-Crebh) on hepatic SREBP-1c production and lipogenesis in vitro and in vivo were investigated. We also examined whether downregulation of endogenous hepatic Crebh by small interfering (si)RNA restores the fenofibrate effect on hepatic lipogenesis and SREBP-1c production. Finally, we examined the mechanism by which CREBH inhibits hepatic SREBP-1c production. Results Fasting and fenofibrate treatment induced CREBH production and decreased SREBP-1c levels. Indeed, AdCrebh inhibited insulin- and liver $\mathrm{X}$ receptor agonist TO901317-induced Srebp-1c (also known as Srebf1) mRNA
\end{abstract}

Electronic supplementary material The online version of this article (doi:10.1007/s00125-012-2771-2) contains peer-reviewed but unedited supplementary material, which is available to authorised users.

A.-K. Min and J. Y. Jeong contributed equally to this study.

A.-K. Min • J. Y. Jeong • Y. Go • Y.-K. Choi • Y.-D. Kim •

I.-K. Lee $(\bowtie) \cdot$ K.-G. Park $(\bowtie)$

Division of Endocrinology and Metabolism,

Department of Internal Medicine,

Research Institute of Aging and Metabolism, WCU Program,

Kyungpook National University School of Medicine,

Daegu 700-721, South Korea

e-mail: leei@knu.ac.kr

e-mail:kpark@knu.ac.kr expression in cultured hepatocytes. Moreover, increased production of CREBH in the liver of mice following tail-vein injection of Ad-Crebh inhibited high-fat diet-induced hepatic steatosis through inhibition of Srebp-1c expression. The inhibition of endogenous $\mathrm{Crebh}$ expression by siRNA restored fenofibrate-induced suppression of Srebp-1c expression and hepatic lipid accumulation both in vitro and in vivo.

Conclusions/interpretation These results show that fenofibrate decreases hepatic lipid synthesis through induction of CREBH. This study suggests CREBH as a novel negative regulator of SREBP-1c production and hepatic lipogenesis.

Keywords CREBH $\cdot$ Fenofibrate $\cdot$ Hepatic steatosis · Lipogenesis · SREBP-1c

\begin{tabular}{|c|c|}
\hline \multicolumn{2}{|c|}{ Abbreviations } \\
\hline Ad & Adenovirus \\
\hline ChIP & Chromatin immunoprecipitation \\
\hline CREBH & $\begin{array}{l}\text { Cyclic AMP response element binding } \\
\text { protein H }\end{array}$ \\
\hline ER & Endoplasmic reticulum \\
\hline EMSA & Electrophoretic mobility shift assay \\
\hline FA & Fatty acids \\
\hline GFP & Green fluorescent protein \\
\hline HFD & High-fat diet \\
\hline IPGTT & Intraperitoneal glucose tolerance test \\
\hline LXR & Liver X receptor \\
\hline MOI & Multiplicity of infection \\
\hline pfu & Plaque-forming units \\
\hline $\operatorname{PPAR} \alpha$ & Peroxisome proliferator-activated receptor $\alpha$ \\
\hline SCAP & SREBP-2 chaperone \\
\hline SD & Sprague-Dawley \\
\hline si & Small interfering \\
\hline SP1 & Specific protein 1 \\
\hline SREBP-1c & Sterol regulatory element binding protein- $1 \mathrm{c}$ \\
\hline STZ & Streptozotocin \\
\hline
\end{tabular}




\section{Introduction}

Non-alcoholic fatty liver disease, an important hepatic manifestation of metabolic syndrome, is a significant cause of morbidity and mortality in obese individuals with insulin resistance $[1,2]$. The onset of fatty liver disease is characterised by increased intracellular triacylglycerol accumulation, causing a condition known as 'fatty liver'. Sterol regulatory element binding protein-1c (SREBP-1c) is a major regulator of the expression of genes involved in hepatic triacylglycerol synthesis [3]. Its role in the development of fatty liver in obese individuals with insulin resistance is now well established. Briefly, hyperinsulinaemia, a hallmark of insulin resistance [4], stimulates hepatic SREBP-1c production, resulting in increased de novo synthesis of fatty acids (FA) [5].

The regulation of hepatic SREBP-1c production is largely dependent on nutritional or hormonal stimuli mediated by transcriptional and post-transcriptional mechanisms of gene expression. During feeding, insulin secreted from pancreatic beta cells increases SREBP-1c production through cleavage of precursor SREBP-1c into its active nuclear form and through activation of liver $\mathrm{X}$ receptor (LXR) and specific protein 1 (SP1) $[6,7]$. Although much is known about how hepatic SREBP-1c is induced by insulin, little is known about how hepatic SREBP-1c is suppressed during fasting, when the effect of insulin is negligible. It is generally accepted that glucagon is the major inhibitor of lipogenesis during the fasting state, because of its activation of the cAMP/protein kinase A pathway $[8,9]$. However, the mechanism of action of protein kinase $\mathrm{A}$ is not well defined [10]. Moreover, the mechanism by which the absence of insulin suppresses hepatic lipogenesis remains to be elucidated. Pharmacological agents that inhibit transcription factors regulating SREBP-1c production during insulin stimulation may provide effective treatment options for fatty liver disease.

Fenofibrate is used clinically to treat hypertriacylglycerolaemia in patients with metabolic syndrome [11]. Fenofibrate lowers serum triacylglycerol levels through several mechanisms, including increasing VLDL-triacylglycerol clearance and decreasing hepatic triacylglycerol production [12]. Hepatic VLDL production depends on the availability of FA, which is determined by de novo FA synthesis and $\beta$-oxidation of FA in the liver [13]. Fenofibrate interferes with FA synthesis and stimulates hepatic fatty acid oxidation, thereby reducing the amount of FA available to the liver for triacylglycerol synthesis [14-16]. Accumulating evidence indicates that the pharmacological actions of fenofibrate are mediated, at least in part, through the transcriptional regulation of several genes involved in lipid and lipoprotein metabolism [17, 18]. Fenofibrate activates the transcriptional factor peroxisome proliferator-activated receptor $\alpha(\operatorname{PPAR} \alpha)$, which binds to peroxisome proliferator response elements in the regulatory regions of target genes [19]. PPAR $\alpha$ mediates fenofibratemediated regulation of triacylglycerol levels via the transcriptional induction of lipoprotein lipase [20]. However, few studies have investigated the direct role of fenofibrate in SREBP-1c transcriptional regulation during hepatic lipogenesis.

Cyclic AMP response element binding protein $\mathrm{H}$ (CREBH) is a membrane-bound transcription factor possessing a basic leucine zipper (bZIP) domain and a domain that resides within the endoplasmic reticulum (ER) [21, 22]. CREBH is cleaved on ER stress and activates the expression of acute response genes [23]. A recent study showed that CREBH is nutritionally regulated [24]. The expression of Crebh (also known as Creb313) mRNA and levels of CREBH protein are high in the fasting mouse liver and are suppressed by re-feeding. The authors also found that feeding-induced suppression of CREBH depends on insulin. Recently, a role for CREBH in triacylglycerol metabolism has been suggested. Genetically CREBH-deficient mice showed higher plasma triacylglycerol concentrations than wild-type mice, and this was due to inefficient triacylglycerol clearance catalysed by lipoprotein lipase [25]. More recently, Zhang et al showed that disruption of CREBH activity leads to massive accumulation of hepatic lipid metabolites and significant increases in plasma triacylglycerol levels in animals fed with an atherogenic high-fat diet (HFD) [26]. However, the role of CREBH in controlling insulin- and LXR-stimulated SREBP-1c production and hepatic lipogenesis by a pharmacological lipid-lowering agent remains to be elucidated. Here, we investigated whether CREBH mediates fenofibrate-induced suppression of hepatic lipogenesis by inhibiting insulin- and LXR-stimulated SREBP-1c production.

\section{Methods}

Materials and plasmids

Insulin was provided by Novo Nordisk (Bagsvaerd, Denmark). T0901317 and fenofibrate were purchased from Sigma (St Louis, MO, USA). The SREBP-1c antibody was purchased from BD Biosciences (San Jose, CA, USA), and the actin antibody was from Sigma. Radiochemicals (deoxycytidine $5^{\prime}-\left[\alpha-{ }^{32} \mathrm{P}\right]$ triphosphate and adenosine $5^{\prime}-\left[\gamma_{-}{ }^{32} \mathrm{P}\right]$ triphosphate) were purchased from Perkin Elmer (Boston, MA, USA). Constructs of human SREBP-1c (also known as SREBF1)-promoter-luciferase (pSREBP-1c[-1516/+40]luciferase) were kind gifts from L. Cagen (University of Tennessee, TN, USA). A cDNA encoding a constitutively active form of mouse Crebh (also known as Creb313) were provided by H.-S. Choi (Chonnam National University, 
Gwangju, Korea). Human INSIG2-promoter-reporter constructs was provided by M. Casado (Instituto de Biomedicina de Valencia, Barcelona, Spain).

\section{Cell culture}

Rat hepatoma H4IIE and human hepatoma HepG2 cells and mouse hepatocyte AML-12 cells were cultured in medium and infected with Ad-Crebh. For details of cell culture, refer to the electronic supplementary material (ESM) Methods.

Generation of recombinant adenovirus For details of the preparation of recombinant adenovirus, refer to the ESM Methods.

Chromatin immunoprecipitation assay To investigate the binding of protein to promoter, chromatin immunoprecipitation (ChIP) assays were done. For details of the ChIP assay, refer to the ESM Methods.

Animal experiments

Experimental procedure 1 All animal procedures were approved by the local ethics committee and carried out in accordance with institutional guidelines for animal research. Male 8-week-old Sprague-Dawley (SD) rats weighing 250 to $300 \mathrm{~g}$ were divided into two groups: fasting and feeding. Animals were fasted for $24 \mathrm{~h}$ with free access to water or fasted for $24 \mathrm{~h}$ and re-fed during the following $12 \mathrm{~h}$. To demonstrate that feeding-induced hepatic SREBP-1c production is insulin dependent, we performed an additional experiment employing intraperitoneal administration of streptozotocin (STZ; $100 \mathrm{mg} / \mathrm{kg}$ ) to eliminate endogenous insulin. STZ-treated rats were divided into two groups, fasting and feeding, and subjected to the fasting/feeding protocol described above.

Experimental procedure 2 Male 8-week-old C57BL/6 mice were purchased from Koatech (Kyunggi-Do, Korea) and divided into five groups: (1) animals fed control chow diet; (2) animals fed an HFD that provided $60 \%$ of energy as fat (D12492; Research Diets, New Brunswick, NJ, USA) alone; (3) HFD-fed group mice infected with adenoviral vector expressing Crebh $\left(1 \times 10^{9}\right.$ plaque-forming units [pfu] $)$ by tail-vein injection; (4) animals fed an HFD with fenofibrate (100 mg kg ${ }^{-1} \mathrm{day}^{-1}$ ); and (5) animals fed an HFD with fenofibrate $\left(100 \mathrm{mg} \mathrm{kg}^{-1} \mathrm{day}^{-1}\right)$ and infected with adenoviral vector expressing small interfering (si)RNA Crebh $\left(1 \times 10^{9} \mathrm{pfu}\right)$ by tail-vein injection at the indicated time points. After 4 weeks, animals were killed for the collection of blood and liver samples.
Histological analysis Hepatic lipid accumulation was examined by histological analysis. See the ESM Methods.

Intraperitoneal glucose tolerance test The effect of fenofibrate on insulin sensitivity was determined by intraperitoneal glucose tolerance test (IPGTT). For details of the IPGTT, refer to the ESM Methods.

Analysis of blood samples Blood levels of glucose, insulin, triacylglycerol and NEFA were measured in overnightfasted mice. For details of the analyses, refer to the ESM Methods.

Genetic analyses in cells and tissues

Quantitative real-time RT-PCR To quantify the DNA in cells and tissues, quantitative real-time RT-PCR was used. For details, refer to the ESM Methods.

Northern blot analysis Northern blot analysis was used to measure mRNA expression. For details, refer to the ESM Methods.

Western blot analysis Protein levels in cells and tissues were examined by western blot analysis. For details of western blotting procedures, refer to the ESM Methods.

In vitro transient transfections and reporter assays Promoter activity was determined by in vitro transfection and luciferase reporter assays. For details, refer to the ESM Methods.

Electrophoretic mobility shift assay DNA-binding activity was measured using an electrophoretic mobility shift assay (EMSA). For details, refer to the ESM Methods.

Statistical analysis

Data were evaluated using ANOVA followed by a post-hoc least-significant-difference test, and expressed as the mean \pm SEM. Values of $p<0.05$ were considered statistically significant. All experiments were repeated independently three or more times.

\section{Results}

Fasting and fenofibrate treatment increase $\mathrm{Crebh}$ expression but decrease Srebp-1c expression

Insulin is one of the most important hormones involved in regulating hepatic lipogenesis. In cultured hepatocytes, insulin increases lipogenesis primarily through the induction 
of SREBP-1c [27]. In addition, re-feeding after restriction of energy intake increases lipogenesis and SREBP-1c production in the liver [28]. To demonstrate that CREBH production is influenced by insulin, we analysed hepatic Crebh expression during fasting and after re-feeding of control and STZ-induced diabetic rats. Re-feeding after fasting dramatically decreased hepatic Crebh mRNA expression and increased Srebp-1c mRNA expression in control rats (Fig. 1a). However, after eliminating endogenous insulin by STZ, re-feeding did not suppress Crebh mRNA expression, suggesting that re-feeding-induced suppression of Crebh mRNA expression is mainly mediated by insulin (Fig. 1b). In addition, no feeding-induced changes in Srebp-1c mRNA expression were observed in the livers of

a

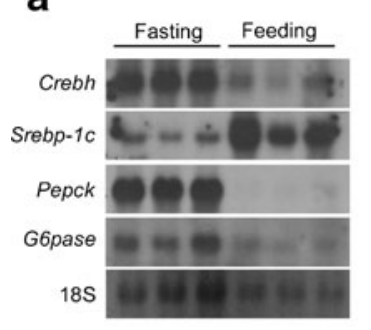

C

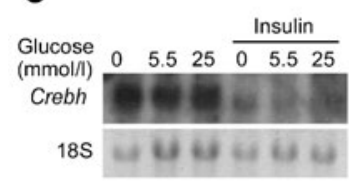

d

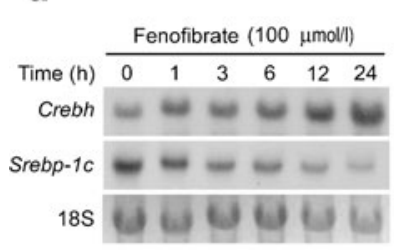

b
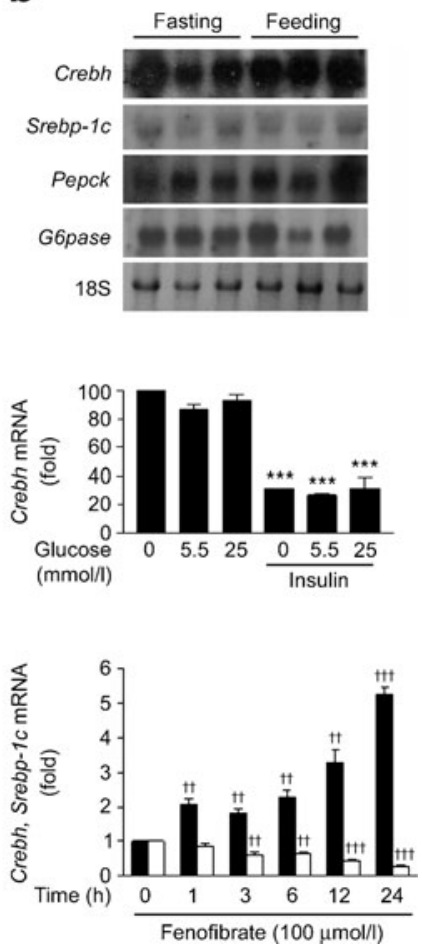

Fig. 1 Fasting and fenofibrate treatment increase $C r e b h$ expression but decrease Srebp-1c expression. (a) Representative northern blot analysis of hepatic Crebh and Srebp-1c mRNA expression in the livers of normal SD rats undergoing fasting and re-feeding. Animal experiments were performed as described in the Methods. (b) Representative northern blot analysis of hepatic Crebh and Srebp-1c mRNA expression in STZ-treated SD rats with fasting and re-feeding. (c) Representative northern blot analysis of Crebh mRNA expression in the presence of insulin. H4IIE cells were incubated with the indicated concentrations of glucose for $24 \mathrm{~h}$, with or without treatment by incubation with $100 \mathrm{nmol} / 1$ insulin for $6 \mathrm{~h}$. Data represent the mean \pm SE of three independent measurements; ${ }^{* * *} p<0.001$ vs glucose alone. (d) Representative northern blot analysis of Crebh and Srebp- $1 c$ mRNA expression in the presence of fenofibrate. H4IIE cells were incubated with fenofibrate $(100 \mu \mathrm{mol} / 1)$ for the indicated times. Data represent the mean $\pm \mathrm{SE}$ of three independent measurements. ${ }^{\dagger \dagger} p<0.01$ and ${ }^{\dagger \dagger} p<0.001$ vs control. Black bar, Crebh; white bar, Srebp-1c. G6pase is also known as G6pc; Pepck is also known as Pck1
STZ-treated rats (Fig. 1b). The expression patterns of Srebp$1 c$ were opposite to those of Crebh. The effect of insulin on Crebh expression was further confirmed in H4IIE cells. Glucose concentration did not influence Crebh mRNA expression, and insulin treatment significantly reduced $\mathrm{Crebh}$ mRNA expression (Fig. 1c). Collectively, these data suggest that insulin is a major positive regulator of hepatic SREBP-1c production, but a negative regulator of CREBH production. Because fenofibrate is used clinically for lowering hepatic triacylglycerol synthesis, which is induced by hyperinsulinaemia, we evaluated the effect of fenofibrate on CREBH and SREBP-1c production in H4IIE cells. Interestingly, fenofibrate increased $\mathrm{Crebh}$ mRNA expression but decreased Srebp-1c mRNA expression (Fig. 1d). Accordingly, fenofibrate decreased the expression of SREBP-1c target genes, including acetyl-CoA carboxylase $(A c c)$, fatty acid synthase (Fas [also known as Fasn]), and stearoyl-CoA desaturase (Scd [also known as Scd1]) (ESM Fig. 1a). Moreover, siRNA knockdown of CREBH prevented the inhibitory effect of fenofibrate on Srebp-1c expression (ESM Fig. 1b). Taken together with the results of the insulin analyses, fenofibrate increases $\mathrm{CREBH}$ production but decreases SREBP-1c production, which suggests that Srebp-1c may be a CREBH target gene and that CREBH mediates the effects of fenofibrate on hepatic lipogenesis through inhibition of SREBP-1c production induced by hyperinsulinaemia.

CREBH inhibits insulin- and LXR-stimulated SREBP-1c production in cultured hepatocytes

To elucidate the association between CREBH and insulininduced hepatic lipogenesis, we examined whether adenovirus-mediated overexpression of Crebh inhibits insulin-stimulated SREBP-1c production in cultured hepatocytes. As shown in Fig. 2a,b, insulin treatment increased Srebp-1c mRNA expression and precursor and nuclear SREBP-1c protein production. However, insulin-stimulated Srebp-1c mRNA expression and SREBP-1c protein production were inhibited by Ad-Crebh in a dose-dependent manner. Accordingly, Ad-Crebh inhibited the insulin-stimulated expression of SREBP-1c-target-gene mRNA (Acc, Fas and $S c d$; Fig. 2c, d, e). Transient transfection with the human SREBP-1c promoter reporter [29] in HepG2 cells showed that CREBH inhibits insulin-stimulated SREBP-1c mRNA expression at the transcriptional level (Fig. 2f). Next, to determine if CREBH directly binds to the $S R E B P-1 c$ promoter to inhibit its activity, we performed a ChIP assay of the putative CREBH binding sites [30] $(-400 /-394$ and $-318 /-312)$ in the SREBP$1 c$ promoter. $\mathrm{CREBH}$ did not bind to these putative binding sites (data not shown). However, it is possible that CREBH directly binds to other regions of SREBP-1C promoter that were not examined in this study. 
a
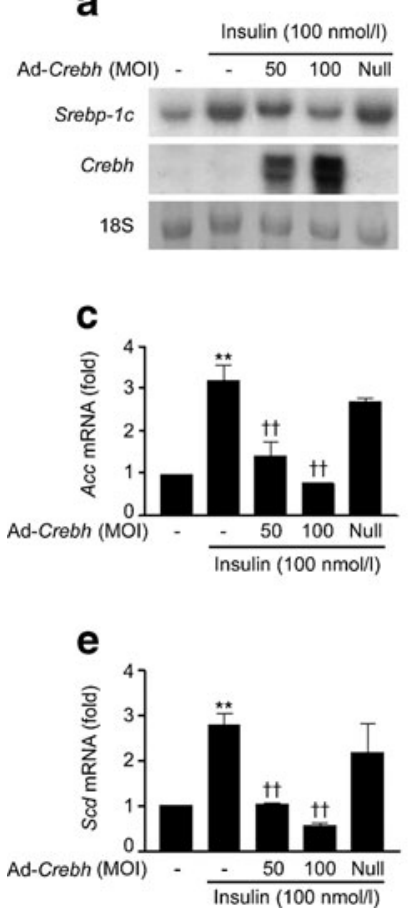

b
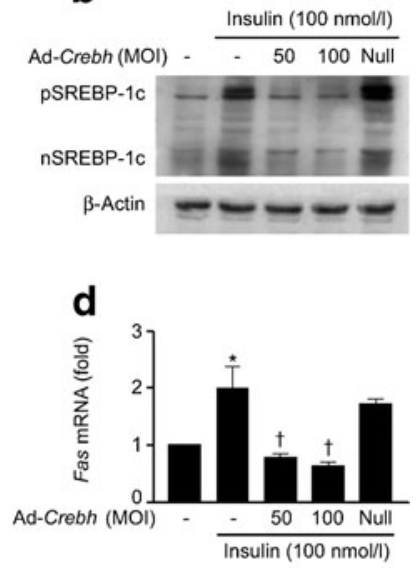

$\mathbf{f}$

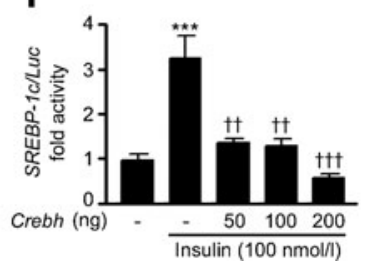

Fig. 2 CREBH inhibits insulin-stimulated SREBP-1C production in cultured hepatocytes. (a) Representative northern blot and (b) western blot analysis of CREBH on insulin-stimulated Srebp-1c mRNA expression and SREBP-1c protein production in H4IIE cells. Cells were infected with the indicated doses (multiplicity of infection [MOI]) of Ad-Crebh or Ad-null for $24 \mathrm{~h}$ and treated with insulin $(100 \mathrm{nmol} / \mathrm{l})$ for $6 \mathrm{~h}$. The ' $\mathrm{p}$ ' and ' $\mathrm{n}$ ' denote the precursor and cleaved nuclear forms of SREBP, respectively. (c-e) Representative real-time RT-PCR analysis of (c) Acc, (d) Fas and (e) Scd mRNA expression in H4IIE cells. Cells were infected with the indicated doses (MOI) of Ad-Crebh or Ad-null for $24 \mathrm{~h}$ and treated with insulin $(100 \mathrm{nmol} / \mathrm{l})$ for $6 \mathrm{~h}$. Data represent the mean \pm SE of three independent measurements. ${ }^{*} p<0.05$ and ${ }^{* *} p<0.01$ vs control; ${ }^{\dagger} p<0.05$ and ${ }^{\dagger} p<0.01$ vs insulin alone. (f) The effect of CREBH on insulin-stimulated SREBP-1c promoter activity. HepG2 cells were cotransfected with $\mathrm{p} S R E B P-1 c(-1516 /+40)$-luciferase construct $(200 \mathrm{ng} / \mathrm{well})$ and the indicated amounts of an expression vector for $\mathrm{Crebh}$, and then stimulated with insulin for $6 \mathrm{~h}$. Data represent the mean \pm SE of three independent measurements. ${ }^{* * *} p<0.001$ vs control; ${ }^{\dagger \dagger} p<0.01$ and ${ }^{\dagger \dagger} p<0.001$ vs insulin alone

LXR plays a major role in regulating SREBP-1c induction, especially on insulin stimulation [29]. Thus, we examined whether CREBH inhibits LXR-stimulated SREBP-1c production. Indeed, Ad-Crebh inhibited TO901317stimulated Srebp-1c mRNA expression and SREBP-1c protein production in a dose-dependent manner (Fig. 3a, b). Ad-Crebh also suppressed TO901317-stimulated Acc, Fas and $S c d$ mRNA expression (Fig. 3c, d, e). To examine the mechanism underlying CREBH-mediated inhibition of LXR activity, we first tested whether Ad-Crebh influenced the abundance of LXR protein. Western blot analysis showed that Ad-Crebh did not decrease LXR protein production (ESM Fig. 2). Next, we examined whether CREBH influences LXR transcriptional activity on the SREBP-1c

promoter. Transient transfection with a promoter reporter construct showed that TO901317 induced a marked increase in SREBP-1c promoter activity, which was inhibited by Crebh (Fig. 3f). Moreover, TO901317 induced an increase in the DNA-binding activity of LXR, which was inhibited by Ad-Crebh in a dose-dependent manner (Fig. 3g). To further confirm that CREBH inhibits LXR binding to LXR response element within the $S R E B P-1 c$ promoter, we performed a ChIP assay. In agreement with the EMSA assay, the results showed that TO901317 induced binding of LXR to the proximal region, which contains two LXR-binding sites. Ad-Crebh abolished the DNA-binding activity of LXR to the SREBP-1c promoter (Fig. 3h).

Fenofibrate inhibits SREBP-1c production through induction of CREBH production

Next, we examined whether fenofibrate inhibits SREBP-1c production and whether fenofibrate-induced CREBH production mediates SREBP-1c production. Treatment with fenofibrate for $24 \mathrm{~h}$ dose-dependently attenuated insulin- or TO901317-stimulated Srebp-1c mRNA expression (Fig. 4a, b) and SREBP-1c protein production (Fig. 4c, d) in H4IIE cells. Fenofibrate also suppressed insulin- and TO901317stimulated Acc, Fas and Scd mRNA expression (ESM Fig. 3). The induction of Crebh expression by fenofibrate was then examined by real-time RT-PCR (ESM Fig. 3). To further confirm whether the suppression of Srebp-1c gene expression by fenofibrate is mediated by CREBH, we downregulated endogenous $\mathrm{Crebh}$ expression by transfecting H4IIE cells with siRNA Crebh and then treated them with fenofibrate. siRNA Crebh, but not control siRNA, significantly restored fenofibrate-induced suppression of Srebp-1c expression stimulated by TO901317 (Fig. 4e). Because fenofibrate induces fatty acid oxidation [31], we investigated whether CREBH mediates the expression of genes related to fenofibrate-induced fatty acid oxidation. The expression of peroxisome proliferator-activated receptor (PPAR)- $\gamma$ coactivator-1 $\alpha$ (Pgc-1 $\alpha$ [also known as Ppargcla]), carnitine palmitoyltransferase 1 (Cpt-1 [also known as $C p t 1 b]$ ), and Ppara (also known as Ppara) was modestly increased by Ad-Crebh (ESM Fig. 4a). However, fenofibrate markedly increased the expression of these genes (ESM Fig. 4b). Moreover, knockdown of CREBH by siRNA induced a $\sim 25 \%$ reduction in fenofibrate induction of $P g c-1 \alpha$ and $C p t-1$ expression, although it significantly blocked fenofibrate-induced Ppar $\alpha$ expression (ESM Fig. 4b).

Fenofibrate and Ad-Crebh prevent insulin-induced inhibition of Insig2 expression

Because insulin-stimulated transcriptional activity of SREBP$1 \mathrm{c}$ on $S r e b p-1 c$ gene expression is mediated by downregulation 

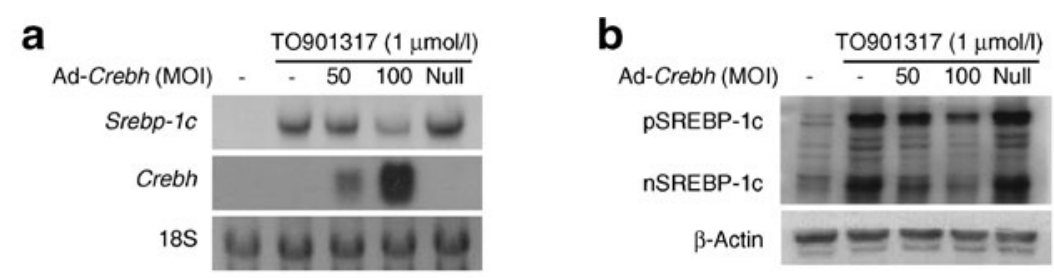
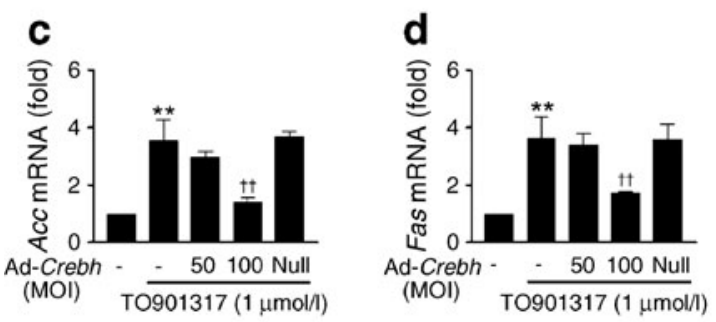

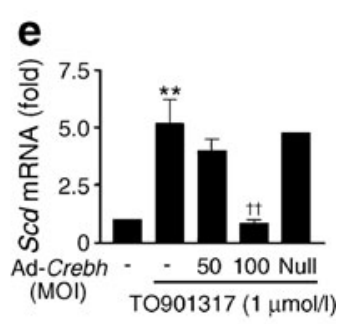

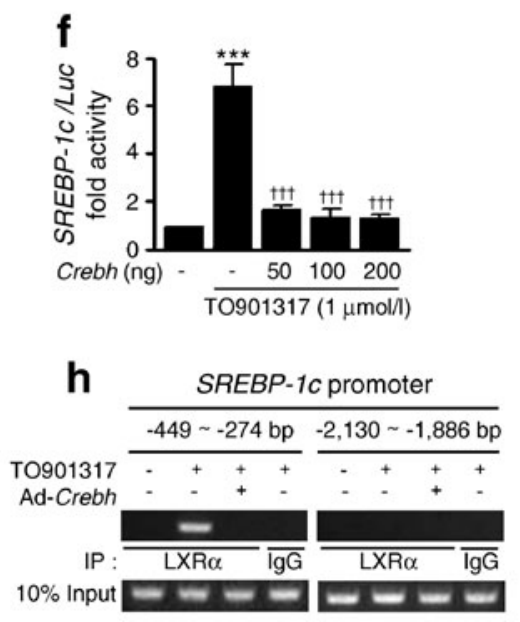

Fig. 3 CREBH inhibits TO901317-stimulated SREBP-1c production and LXR activity. (a) Representative northern blot and (b) western blot analysis of the effect of CREBH on TO901317-stimulated Srebp-1c mRNA expression and SREBP-1c protein production in H4IIE cells. Cells were infected with the indicated doses (MOI) of Ad-Crebh or Adnull for $24 \mathrm{~h}$ and treated with TO901317 (1 $\mu \mathrm{mol} / \mathrm{l})$ for $24 \mathrm{~h}$. The 'p' and ' $n$ ' denote the precursor and cleaved nuclear forms of SREBP-1c, respectively. (c-e) Representative real-time RT-PCR analysis of Acc, $F a s$ and $S c d$ mRNA expression in H4IIE cells. Cells were infected with the indicated doses (MOI) of Ad-Crebh or Ad-null (100 MOI) for $24 \mathrm{~h}$ and treated with TO901317 $(1 \mu \mathrm{mol} / \mathrm{l})$ for $24 \mathrm{~h}$. Data represent the mean \pm SE of three independent measurements. ${ }^{* *} p<0.01$ vs control; ${ }^{\dagger} p<0.01$ vs TO901317 alone. (f) The effect of CREBH on TO901317-

of Insig2 levels, we examined whether fenofibrate and CREBH prevent insulin-suppressed Insig2 expression. As shown Fig. 5a, insulin and Ad-Crebh did not influence Insig1 mRNA expression but fenofibrate markedly increased Insig1 expression; INSIG1 is dissociated from SREBP-2 chaperone (SCAP)-SREBP-2 under the conditions of sterol depletion, consequently causing SCAP-SREBP-2 complex transportation from the ER to the Golgi. In contrast, insulin decreased but fenofibrate and Ad-Crebh increased Insig2 mRNA expression levels (Fig. 5b). Therefore, we further examined whether siRNA knockdown of endogenous INSIG2 blocked the suppression of $S r e b p-1 c$ expression induced by CREBH and

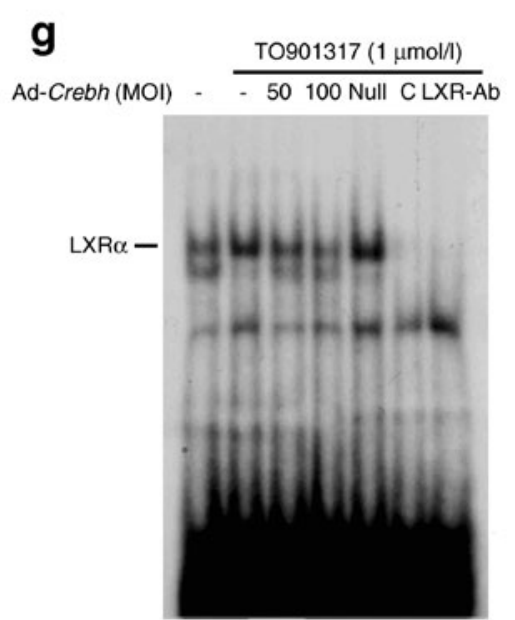

stimulated SREBP-1c promoter activity. HepG2 cells were cotransfected with $S R E B P-1 c$ promoters $(200 \mathrm{ng} / \mathrm{well})$ and the indicated amounts of an expression vector for Crebh and then stimulated with TO901317 $(1 \mu \mathrm{mol} / \mathrm{l})$ for $24 \mathrm{~h}$. Data represent the mean \pm SE of three independent measurements. ${ }^{* * *} p<0.001$ vs control; ${ }^{\dagger \dagger \dagger} p<0.001 \mathrm{vs}$ TO901317 alone. (g) The effect of CREBH on TO901317-stimulated LXR-DNA binding activity. HepG2 cells were infected with the indicated doses (MOI) of Ad-Crebh or Ad-null for $24 \mathrm{~h}$ and treated with TO901317 (1 $\mu \mathrm{mol} / \mathrm{l})$ for $24 \mathrm{~h}$. (h) A ChIP assay was performed using an anti-LXR $\alpha$ antibody. HepG2 cells were infected with the indicated MOI of Ad-Crebh or Ad-null (100 MOI) for $24 \mathrm{~h}$ and treated with TO901317 (1 $\mu \mathrm{mol} / \mathrm{l})$ for $24 \mathrm{~h}$. C, control; IP, immunoprecipitation target

fenofibrate. The results showed that Insig2 siRNA markedly inhibited endogenous Insig2 expression, and knockdown of INSIG2 significantly abolished Ad-Crebh- and fenofibratemediated suppression of insulin-stimulated Srebp-1c expression (Fig. 5c, d). To determine whether expression of Insig2 was transcriptionally regulated by $\mathrm{CREBH}$, we next examined the effects of CREBH on INSIG2 promoter activity. Indeed, a transient transfection study with INSIG2 promoter luciferase [32] showed that Crebh markedly increased INSIG2 promoter $(-859 /-11$ construct) activity, but this activation was markedly attenuated by truncation at -688 bp of the INSIG 2 promoter, suggesting that CREBH mainly acts on the region between 

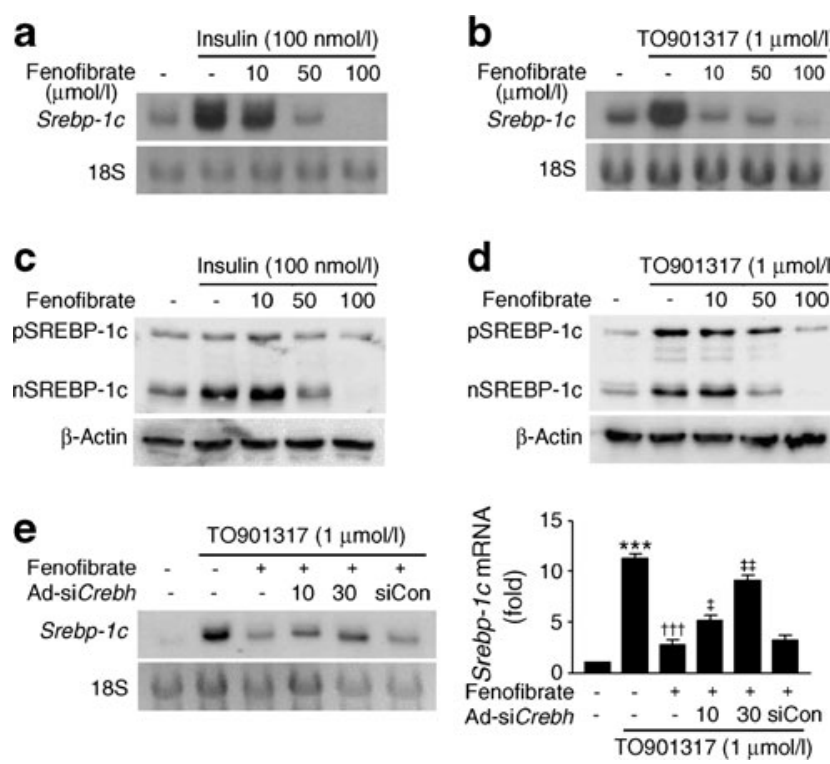

Fig. 4 Fenofibrate inhibition of SREBP-1c production is partially mediated by CREBH. (a,b) Representative northern blot and (c,d) western blot analysis of the effect of fenofibrate on insulin $(\mathbf{a}, \mathbf{b})$ or TO901317 (c,d)-stimulated Srebp-1c mRNA expression and SREBP1c protein production in H4IIE cells. Cells were incubated with insulin $(100 \mathrm{nmol} / \mathrm{l})$ for $6 \mathrm{~h}$ or TO901317 $(1 \mu \mathrm{mol} / \mathrm{l})$ for $24 \mathrm{~h}$, with or without pretreatment with the incubated concentrations of fenofibrate for $24 \mathrm{~h}$. The ' $p$ ' and ' $n$ ' denote the precursor and cleaved nuclear forms of SREBP-1c, respectively. (e) Representative northern blot analysis of siRNA Crebh on the fenofibrate decreased TO901317-stimulated Srebp-1c mRNA expression in H4IIE cells. Cells were infected with the indicated doses (MOI) of Ad-siRNA Crebh or Ad-siRNA control for $24 \mathrm{~h}$ and then treated with fenofibrate $(100 \mu \mathrm{mol} / \mathrm{l})$ and insulin for $6 \mathrm{~h}$ or TO901317 $(1 \mu \mathrm{mol} / \mathrm{l})$ for $24 \mathrm{~h} .{ }^{* * *} p<0.001 \mathrm{vs}$ control; ${ }^{\dagger \dagger \dagger} p<$ 0.001 vs TO901317 alone; ${ }^{\star} p<0.05$ and ${ }^{\star *} p<0.01$ vs TO901317 plus fenofibrate with or without control siRNA. siCrebh, siRNA Crebh; siCon, Ad-siRNA control

-859 and -688 of the INSIG2 promoter to activate INSIG2 promoter activity (ESM Fig. 5). These results suggest that fenofibrate and CREBH retained SREBP-1c in the ER membrane. However, in addition to the increase in Insigl expression, the effect of fenofibrate on INSIG2 production was much greater than that of Ad-Crebh, suggesting a CREBHindependent pathway.

CREBH reduces hepatic lipid accumulation and SREBP-1c production and mediates fenofibrate inhibition of hepatic lipid accumulation in HFD-fed mice

Finally, we examined whether CREBH decreases hepatic lipogenesis and mediates fenofibrate-suppressed hepatic lipogenesis in HFD-fed mice. Histological examination showed that hepatocytes of HFD-fed mice were distended by large cytoplasmic lipid droplets. This change in cellular morphology was nearly completely prevented by the overexpression of Crebh and fenofibrate treatment (Fig. 6a). Both the overexpression of $\mathrm{Crebh}$ and fenofibrate treatment
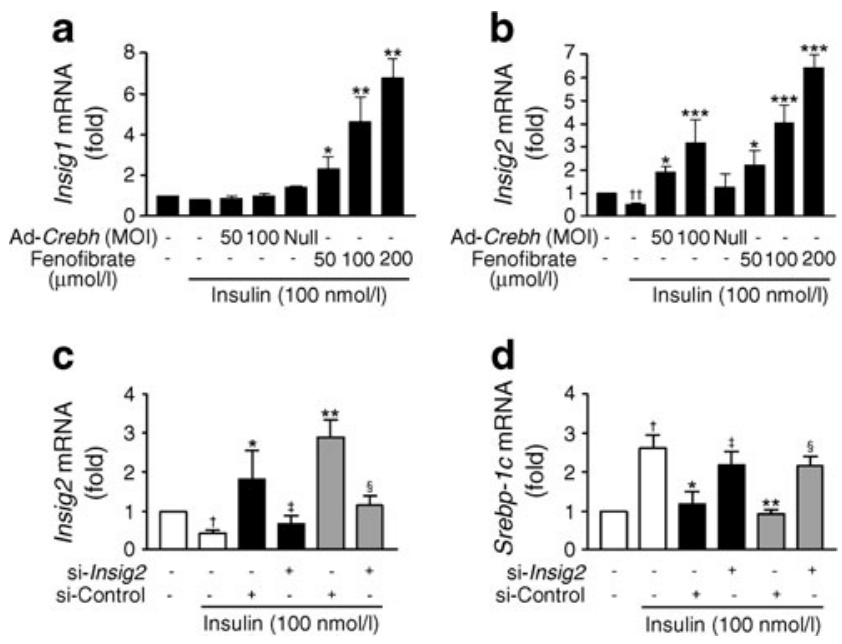

Fig. 5 Fenofibrate and Ad-Crebh inhibit insulin-induced inhibition of Insig2 expression. (a) Representative real-time RT-PCR analysis of Insig1 mRNA expression in H4IIE cells. Cells were infected with the indicated doses (MOI) of Ad-Crebh or incubated concentrations of fenofibrate for $24 \mathrm{~h}$ and treated with insulin $(100 \mathrm{nmol} / \mathrm{l})$ for $6 \mathrm{~h}$. Data represent the mean $\pm \mathrm{SE}$ of three independent measurements. ${ }^{*} p<0.05$ and ${ }^{* *} p 0.01$ vs insulin alone. (b) Representative real-time RT-PCR analysis of Insig 2 mRNA expression in H4IIE cells. Cells were infected with the indicated doses (MOI) of Ad-Crebh or incubated concentrations of fenofibrate for $24 \mathrm{~h}$ and treated with insulin $(100 \mathrm{nmol} / \mathrm{l})$ for $6 \mathrm{~h}$. Data represent the mean $\pm \mathrm{SE}$ of three independent measurements. ${ }^{\dagger \dagger} p<0.01$ vs control; ${ }^{*} p<0.05$ and ${ }^{* * *} p<0.001$ vs insulin alone. (c, d) Representative real-time RT-PCR analysis of Insig2 (c) and Srebp-1c (d) mRNA expression. Cells were transfected with siRNA Insig2 or control scramble siRNA for $24 \mathrm{~h}$ and infected with the indicated doses (MOI) of Ad-Crebh, or incubated with the indicated concentrations of fenofibrate for $24 \mathrm{~h}$ and then treated with insulin $(100 \mathrm{nmol} / \mathrm{l})$ for $6 \mathrm{~h}$. Data represent the mean $\pm \mathrm{SE}$ of three independent measurements. ${ }^{*} p<0.05$ and ${ }^{* *} p<0.01$ vs insulin alone; ${ }^{\dagger} p<0.05$ vs control; ${ }^{\star} p<0.05$ vs insulin plus Ad-Crebh with control scramble siRNA; ${ }^{\S} p<0.05$ vs insulin plus fenofibrate with control scramble siRNA. White bars, control; black bars, Ad-Crebh; grey bars, fenofibrate. si-Control, control scramble siRNA; si-Insig2, siRNA Insig2

significantly decreased hepatic fat accumulation and plasma triacylglycerol levels compared with mice infected with an adenovirus encoding green fluorescent protein (GFP; Fig. 6b, c). Moreover, downregulation of endogenous Crebh by Ad-siRNA Crebh significantly blocked fenofibratesuppressed hepatic lipid accumulation and plasma triacylglycerol levels in HFD-fed mice (Fig. 6b,c). Consistent with the histological examination, both Ad-Crebh and fenofibrate reduced Srebp-1c expression but increased Insig2 expression (Fig. 6d, e). Moreover, the effects of fenofibrate on hepatic Srebp-1c and Insig2 expression were abolished by Ad-siRNA Crebh (Fig. 6d, e). Fenofibrate treatment increased Crebh expression, but Ad-siRNA Crebh suppressed fenofibrate-induced $\mathrm{Crebh}$ expression to basal levels (Fig. 6f). Because CREBH controls the expression of genes involved in gluconeogenesis, we wanted to know whether fenofibrate treatment influences other metabolic variables. 

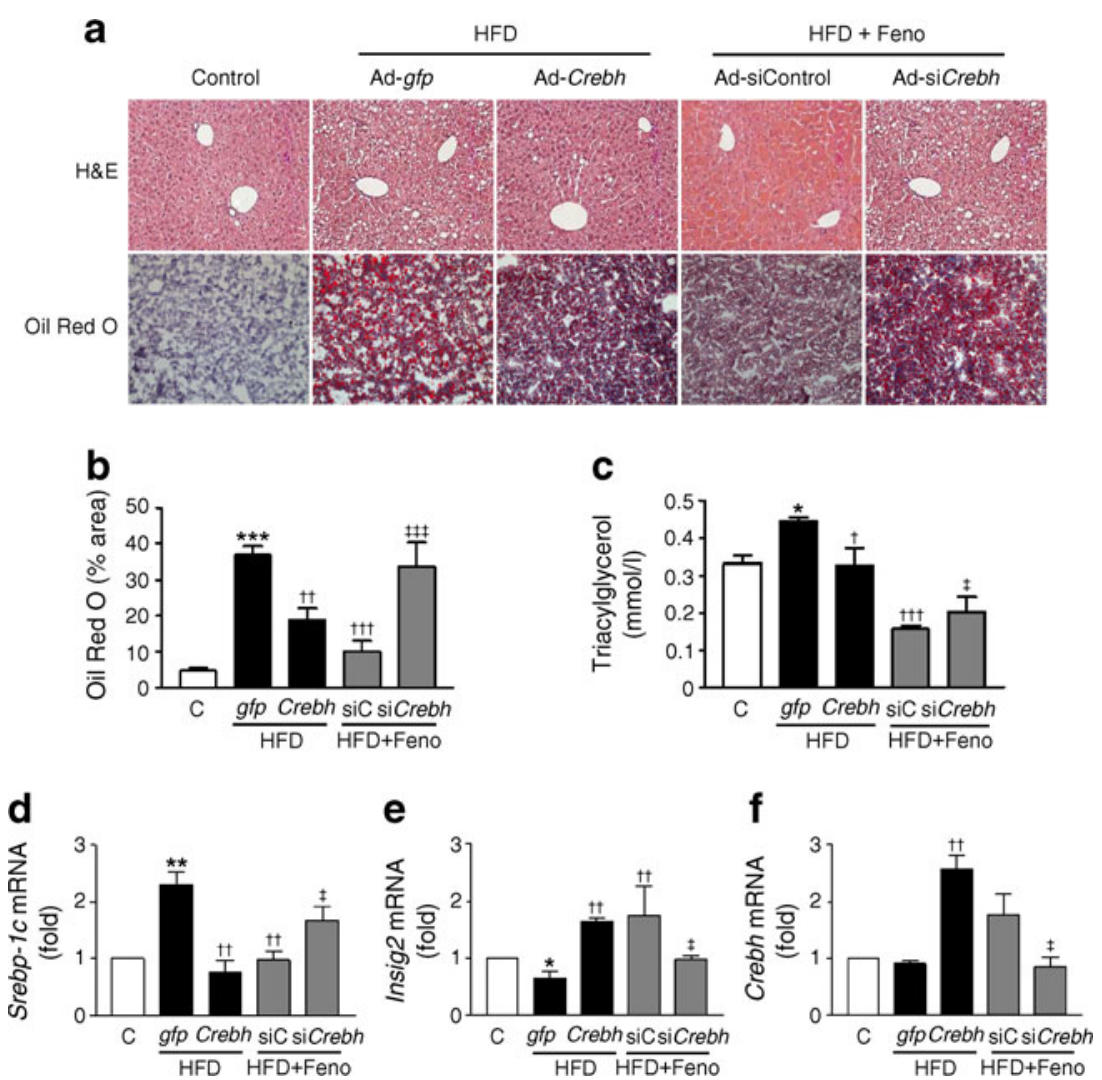

Fig. 6 CREBH reduces hepatic lipid accumulation and SREBP-1c production and mediates fenofibrate inhibition of hepatic lipid accumulation in HFD-fed mice. (a) Histological analysis of liver sections from HFD-fed mice with or without fenofibrate treatment by tail-vein injection of the adenovirus encoding the active form of Crebh, $g f p$, siRNA Crebh and siRNA control. Haematoxylin and eosin staining and Oil Red O staining were used. Original magnification $\times 200$. (b) Morphometric analysis of Oil Red O staining in liver sections from HFD-fed mice with or without fenofibrate treatment by tail-vein injection of the adenovirus encoding the active form of CREBH or GFP, siRNA Crebh or siRNA control. The data represent the mean $\pm \mathrm{SE}$ of three independent measurements ( $n=6$ in each group). ${ }^{* * *} p<0.001 \mathrm{vs}$ control-diet-fed mice; ${ }^{\dagger \dagger} p<0.01$ and ${ }^{\dagger \dagger \dagger} p<0.001$ vs HFD-fed mice;

Plasma glucose, insulin and NEFA levels were lower and insulin sensitivity (IPGTT) was higher in the fenofibratetreated group than in the control HFD group. Serum cholesterol levels were not different between the groups (ESM Fig. 6).

\section{Discussion}

In the present study, we found that fasting and fenofibrate treatment induced CREBH production, while feeding and insulin treatment decreased CREBH production. Interestingly, production of SREBP-1c, which was upregulated by feeding but inhibited by fenofibrate, showed the opposite effects to those of CREBH. Fenofibrate treatment and adenovirus-mediated overexpression of $\mathrm{Crebh}$ inhibited
$\mathrm{N}_{\mathrm{t}} p<0.001$ vs HFD-fed mice with fenofibrate treatment and siRNA control. (c) Analysis of serum triacylglycerol in HFD-fed mice with or without fenofibrate treatment after tail-vein injection of an adenovirus encoding the active forms of CREBH or GFP, siRNA Crebh or siRNA control. ${ }^{*} p<0.05$ vs mice fed control diet; ${ }^{\dagger} p<0.05$ and ${ }^{\dagger \dagger} p<0.001$ vs HFD-fed mice; ${ }^{\star} p<0.05$ vs HFD-fed mice with fenofibrate treatment and siRNA control. (d-f) Representative real-time RT-PCR analysis of the effect of Ad-Crebh on Srebp-1c, Insig2 and Crebh expression. The data represent the mean $\pm \mathrm{SE}$ of three independent measurements $(n=6$ in each group). ${ }^{*} p<0.05$ and ${ }^{* *} p<0.01$ vs control-diet-fed mice; ${ }^{\dagger \dagger} p<0.01$ vs HFD-fed mice; ${ }^{\ddagger} p<0.05$ vs HFD-fed mice with fenofibrate treatment and siRNA control. Feno, fenofibrate; H\&E, haematoxylin and eosin; siC, siRNA control

insulin- and LXR agonist TO901317-stimulated Srebp-1c mRNA expression in cultured hepatocytes. Moreover, overexpression of $\mathrm{Crebh}$ in the liver of mice mediated by tailvein injection of Ad-Crebh inhibited hepatic steatosis through inhibition of SREBP-1c induction. The inhibition of endogenous Crebh expression by siRNAs markedly abolished fenofibrate-induced suppression of Srebp-1c expression and hepatic lipid accumulation. These results show that fenofibrate decreases hepatic lipid synthesis through induction of CREBH. Collectively, this study suggests CREBH as a novel negative regulator of hepatic lipogenesis.

Hepatic lipogenesis is regulated by nutritional status [33]. In mammals, fasting decreases and re-feeding increases hepatic lipid synthesis [29]. Insulin is a potent inducer of hepatic lipogenesis during feeding [7, 29], and this may be transcriptionally mediated by SREBP-1c [28, 34, 35]. In the 
present study, while Crebh expression was downregulated by feeding, this downregulation was not observed when endogenous insulin production was eliminated by STZ treatment. Moreover, in cultured hepatocytes, Crebh expression was inhibited by insulin treatment. These data indicate that feeding-induced suppression of $\mathrm{Crebh}$ expression is regulated by insulin. The expression patterns of insulin-regulated CREBH were opposite to those of SREBP-1c, suggesting that CREBH plays a role in the regulation of SREBP-1c production and hepatic lipogenesis. Indeed, we found that adenovirus-mediated overexpression of Crebh inhibited insulin-stimulated SREBP-1c production.

The role of SREBP-1c in the development of fatty liver in the insulin-resistant state is now well established [36]. Hyperinsulinaemia, a hallmark of insulin resistance, stimulates hepatic Srebp-1c transcription, resulting in increased hepatic fat synthesis and the development of fatty liver. We [37] and others [38] demonstrated the contribution of SREBP-1c to fat accumulation in the insulin-resistant HFD-induced mouse liver [39]. Many studies have focused on searching for molecules that can inhibit SREBP-1c production, thereby preventing the development of fatty liver. For example, metformin and $\alpha$-lipoic acid prevent hepatic steatosis by inhibiting SREBP-1c production [29, 40]. Fenofibrate has beneficial effects on metabolic syndrome by downregulating multiple target genes involved in the pathogenesis of hepatic steatosis [41-43]. Recent studies show that fenofibrate induces CREBH production to a level comparable with that observed under fasting conditions [44]. However, the exact molecular mechanism underlying the anti-hepatic steatosis effect of fenofibrate remains unclear. Thus, we aimed to determine the role of fenofibrate-induced CREBH production in the development of hepatic steatosis in mice with HFD-induced insulin resistance. This study showed that adenovirus-mediated overexpression of Crebh in the liver or treatment of fenofibrate prevented HFDinduced hepatic lipid accumulation and inhibited SREBP-1c production. Moreover, inhibition of endogenous $\mathrm{Crebh}$ expression by siRNA prevented fenofibrate-induced suppression of hepatic steatosis, suggesting that CREBH mediates the effects of fenofibrate on hepatic lipogenesis and is a potential therapeutic target for the treatment of hepatic steatosis induced by hyperinsulinaemia.

Insulin-induced SREBP-1c production is regulated at the transcriptional level. The major regulators of Srebp-1c expression are LXR and SREBP-1c itself. LXR is a member of a nuclear receptor superfamily that regulates the production of key proteins involved in lipid metabolism [45, 46]. LXR increases Srebp-1c expression, leading to increased hepatic triacylglycerol synthesis [29]. In our study, fenofibrate and Ad-Crebh inhibited LXR-ligand-stimulated SREBP-1c production. An in vitro transient transfection study employing promoter-reporter constructs showed that inhibition of LXR ligand-stimulated SREBP-1c production by CREBH is mediated via the inhibition of LXR activity on the SREBP-1c promoter. In addition to $\mathrm{LXR}$, in response to insulin, SREBP-1c production is also mediated by the cleaved nuclear form of SREBP-1c itself, which mediates transactivation on the SREBP-1c promoter [33]. A study by Yellaturu et al clearly shows that insulin enhances SREBP-1c production by inducing cleavage of precursor SREBP-1c to its nuclear form [34]. In the resting state, the SCAP-SREBP1c complex is localised to the ER by INSIG2. However, in response to stimuli, INSIG2 dissociates from the SCAPSREBP-1c complex, thereby facilitating transport of the complex to the Golgi where immature SREBP-1c is cleaved to generate the active form of SREBP-1c [34]. Insulin enhances the rate of turnover of Insig2 mRNA, and insulin-induced depletion of Insig2 promotes proteolysis of SREBP-1c, resulting in active SREBP-1c. Our data show that Ad-Crebh and fenofibrate prevent insulin-suppressed Insig2 gene expression. Moreover, knockdown of INSIG2 expression by siRNA abolished Ad-Crebh- and fenofibratemediated suppression of SREBP-1c. Fenofibrate, however, restored insulin-suppressed Insig2 gene expression to a higher level than did CREBH, suggesting a CREBHindependent mechanism. Taken together, the mechanisms by which CREBH influences hepatic lipogenesis and SREBP-1c production are multifactorial. Our data suggest that CREBH mediates fenofibrate-suppressed hepatic lipogenesis by inhibiting LXR-DNA binding to the SREBP-1c promoter and inducing INSIG2-mediated inactivation of SREBP-1c.

As fenofibrate induces the expression of genes involved in fatty acid oxidation by activating PPAR $\alpha$, induction of these genes is thought to be important for the amelioration of hepatic lipid metabolism. Accordingly, the present study showed that fenofibrate markedly increased the expression of genes involved in fatty acid oxidation. However, we found that although Ad-Crebh modestly increased the expression of these genes, the effect was much smaller than that of fenofibrate. Moreover, knockdown of CREBH production with siRNA did not significantly abolish the fenofibrate-mediated induction of these genes, although a reduction in Crebh expression by siRNA tended to decrease the effects of fenofibrate on gene expression. These data suggest that CREBH-mediated effects of fenofibrateinduced inhibition of hepatic steatosis are mainly due to the inhibition of de novo lipogenesis and not to the augmentation of fatty acid oxidation, although CREBH increases the expression of genes responsible for fatty acid oxidation.

In summary, these data indicate a novel role for CREBH in inhibiting hepatic lipogenesis by fenofibrate. Hepatic steatosis is emerging as the most important cause of chronic liver disease associated with the increasing incidence of 
obesity. Hepatic steatosis can progress to non-alcoholic steatohepatitis, as well as to advanced cirrhosis. Thus, an effective therapy is needed to prevent this condition. In the present study, we showed that fenofibrate-induced $\mathrm{CREBH}$ prevents hepatic steatosis in animals with diet-induced insulin resistance. This suggests that $\mathrm{CREBH}$ may serve as a therapeutic target for preventing the development of fatty liver disease in patients with insulin resistance. To more clearly elucidate the role of CREBH in hepatic lipid synthesis, it would be useful to generate animals in which Crebh expression in the liver is conditionally knocked out.

Acknowledgements We would like to thank H.-S. Choi (Chonnam National University, Gwangju, Korea) for helpful discussion, M. Casado (Instituto de Biomedicina de Valencia, Barcelona, Spain) for the gift of the Insig2 promoter luciferase plasmids, and J.-H. Kim and S.-H. Lee (KT\&G Life Science, Suwon, Korea) for biochemical analysis.

Funding This work was supported by grants from the National Research Foundation (2011-0028659, 2012R1A2A2A01043867, WCU program R32-10064 and Future-based Technology Development program Bio Field 2011-0019449) funded by the Ministry of Education, Science, and Technology and a grant of the Korea Health Technology R\&D Project, Ministry of Health \& Welfare, Republic of Korea (A111345).

Duality of interest The authors declare that there is no duality of interest associated with this manuscript.

Contribution statement A-KM contributed to performance of experiments, analysis and interpretation of data and drafting the manuscript. JYJ contributed to the conception of experiments, acquisition of data, analysis and interpretation of data, and revision of the manuscript. YG contributed to the performance of experiments, analysis and interpretation of data and revision of the manuscript. Y-KC contributed to analysis of data and drafting and revision of the manuscript. Y-DK contributed to the analysis and interpretation of data and drafting the manuscript. I-KL and K-GP contributed to the conception and design of the experiments and the article, drafting, critical review and revision of the manuscript. All authors approved the final version of the manuscript to be published.

\section{References}

1. Marceau P, Biron S, Hould FS et al (1999) Liver pathology and the metabolic syndrome $\mathrm{X}$ in severe obesity. J Clin Endocrinol Metab 84:1513-1517

2. Marchesini G, Brizi M, Morselli-Labate AM et al (1999) Association of nonalcoholic fatty liver disease with insulin resistance. Am J Med 107:450-455

3. Horton JD (2002) Sterol regulatory element-binding proteins: transcriptional activators of lipid synthesis. Biochem Soc Trans 30:1091-1095

4. Zammit VA, Waterman IJ, Topping D, McKay G (2001) Insulin stimulation of hepatic triacylglycerol secretion and the etiology of insulin resistance. J Nutr 131:2074-2077

5. Shimomura I, Bashmakov Y, Horton JD (1999) Increased levels of nuclear SREBP-1c associated with fatty livers in two mouse models of diabetes mellitus. J Biol Chem 274:30028-30032

6. Cagen LM, Deng X, Wilcox HG, Park EA, Raghow R, Elam MB (2005) Insulin activates the rat sterol-regulatory-element-binding protein 1c (SREBP-1c) promoter through the combinatorial actions of SREBP, LXR, Sp-1 and NF-Y cis-acting elements. Biochem J 385:207-216

7. Liang G, Yang J, Horton JD, Hammer RE, Goldstein JL, Brown MS (2002) Diminished hepatic response to fasting/refeeding and liver X receptor agonists in mice with selective deficiency of sterol regulatory element-binding protein-1c. J Biol Chem 277:9520-9528

8. Lu M, Shyy JY (2006) Sterol regulatory element-binding protein 1 is negatively modulated by PKA phosphorylation. Am J Physiol Cell Physiol 290:C1477-C1486

9. Yamamoto T, Shimano H, Inoue N et al (2007) Protein kinase A suppresses sterol regulatory element-binding protein-1C expression via phosphorylation of liver $\mathrm{X}$ receptor in the liver. J Biol Chem 282:11687-11695

10. Girard J, Perdereau D, Foufelle F, Prip-Buus C, Ferre P (1994) Regulation of lipogenic enzyme gene expression by nutrients and hormones. FASEB J 8:36-42

11. Rosenson RS (2008) Fenofibrate reduces lipoprotein associated phospholipase A2 mass and oxidative lipids in hypertriglyceridemic subjects with the metabolic syndrome. Am Heart J 155:499.9-16

12. Staels B, Dallongeville J, Auwerx J, Schoonjans K, Leitersdorf E, Fruchart JC (1998) Mechanism of action of fibrates on lipid and lipoprotein metabolism. Circulation 98:2088-2093

13. Bijland S, Pieterman EJ, Maas AC et al (2010) Fenofibrate increases very low density lipoprotein triglyceride production despite reducing plasma triglyceride levels in APOE*3-Leiden.CETP mice. J Biol Chem 285:25168-25175

14. Kritchevsky D, Tepper SA, Story JA (1979) Influence of procetofen on lipid metabolism in normocholesteremic rats. Pharmacol Res Commun 11:635-641

15. Martin G, Schoonjans K, Lefebvre AM, Staels B, Auwerx J (1997) Coordinate regulation of the expression of the fatty acid transport protein and acyl-CoA synthetase genes by PPARalpha and PPARgamma activators. J Biol Chem 272:28210-28217

16. Schoonjans K, Watanabe M, Suzuki H et al (1995) Induction of the acyl-coenzyme A synthetase gene by fibrates and fatty acids is mediated by a peroxisome proliferator response element in the $\mathrm{C}$ promoter. J Biol Chem 270:19269-19276

17. Schoonjans K, Staels B, Auwerx J (1996) Role of the peroxisome proliferator-activated receptor (PPAR) in mediating the effects of fibrates and fatty acids on gene expression. J Lipid Res 37:907-925

18. Schoonjans K, Staels B, Auwerx J (1996) The peroxisome proliferator activated receptors (PPARS) and their effects on lipid metabolism and adipocyte differentiation. Biochim Biophys Acta 1302:93-109

19. Issemann I, Green S (1990) Activation of a member of the steroid hormone receptor superfamily by peroxisome proliferators. Nature 347:645-650

20. Staels B, Auwerx J (1992) Perturbation of developmental gene expression in rat liver by fibric acid derivatives: lipoprotein lipase and alpha-fetoprotein as models. Development 115:1035-1043

21. Chin KT, Zhou HJ, Wong CM et al (2005) The liver-enriched transcription factor CREB-H is a growth suppressor protein underexpressed in hepatocellular carcinoma. Nucleic Acids Res 33:1859-1873

22. Omori Y, Imai J, Watanabe $M$ et al (2001) CREB-H: a novel mammalian transcription factor belonging to the CREB/ATF family and functioning via the box-B element with a liver-specific expression. Nucleic Acids Res 29:2154-2162

23. Zhang K, Shen X, Wu J et al (2006) Endoplasmic reticulum stress activates cleavage of CREBH to induce a systemic inflammatory response. Cell 124:587-599

24. Foretz M, Guichard C, Ferre P, Foufelle F (1999) Sterol regulatory element binding protein- $1 \mathrm{c}$ is a major mediator of insulin action on the hepatic expression of glucokinase and lipogenesis-related genes. Proc Natl Acad Sci U S A 96:12737-12742 
25. Lee JH, Giannikopoulos P, Duncan SA et al (2011) The transcription factor cyclic AMP-responsive element-binding protein $\mathrm{H}$ regulates triglyceride metabolism. Nat Med 17:812-815

26. Zhang C, Wang G, Zheng Z et al (2012) Endoplasmic reticulumtethered transcription factor cAMP responsive element-binding protein, hepatocyte specific, regulates hepatic lipogenesis, fatty acid oxidation, and lipolysis upon metabolic stress in mice. Hepatology 55:1070-1082

27. Horton JD, Bashmakov Y, Shimomura I, Shimano H (1998) Regulation of sterol regulatory element binding proteins in livers of fasted and refed mice. Proc Natl Acad Sci U S A 95:5987-5992

28. Chen G, Liang G, Ou J, Goldstein JL, Brown MS (2004) Central role for liver X receptor in insulin-mediated activation of Srebp-1c transcription and stimulation of fatty acid synthesis in liver. Proc Natl Acad Sci U S A 101:11245-11250

29. Park KG, Min AK, Koh EH et al (2008) Alpha-lipoic acid decreases hepatic lipogenesis through adenosine monophosphateactivated protein kinase (AMPK)-dependent and AMPKindependent pathways. Hepatology 48:1477-1486

30. Lee MW, Chanda D, Yang J et al (2010) Regulation of hepatic gluconeogenesis by an ER-bound transcription factor, CREBH. Cell Metab 11:331-339

31. Oosterveer MH, Grefhorst A, van Dijk TH et al (2009) Fenofibrate simultaneously induces hepatic fatty acid oxidation, synthesis, and elongation in mice. J Biol Chem 284:34036-34044

32. Fernandez-Alvarez A, Soledad Alvarez M, Cucarella C, Casado M (2010) Characterization of the human insulin-induced gene 2 (INSIG2) promoter: the role of Ets-binding motifs. J Biol Chem 285:11765-11774

33. Kammoun HL, Chabanon H, Hainault I et al (2009) GRP78 expression inhibits insulin and ER stress-induced SREBP-1c activation and reduces hepatic steatosis in mice. J Clin Invest 119:1201-1215

34. Yellaturu CR, Deng X, Park EA, Raghow R, Elam MB (2009) Insulin enhances the biogenesis of nuclear sterol regulatory element-binding protein (SREBP)-1c by posttranscriptional down-regulation of Insig-2A and its dissociation from SREBP cleavage-activating protein (SCAP).SREBP-1c complex. J Biol Chem 284:31726-31734
35. Browning JD, Horton JD (2004) Molecular mediators of hepatic steatosis and liver injury. J Clin Invest 114:147-152

36. Kim YW, Kim YM, Yang YM et al (2010) Inhibition of SREBP1c-mediated hepatic steatosis and oxidative stress by sauchinone, an AMPK-activating lignan in Saururus chinensis. Free Radic Biol Med 48:567-578

37. Deng X, Yellaturu C, Cagen L et al (2007) Expression of the rat sterol regulatory element-binding protein-1c gene in response to insulin is mediated by increased transactivating capacity of specificity protein 1 (Sp1). J Biol Chem 282:17517-17529

38. Oakes ND, Cooney GJ, Camilleri S, Chisholm DJ, Kraegen EW (1997) Mechanisms of liver and muscle insulin resistance induced by chronic high-fat feeding. Diabetes 46:1768-1774

39. Yang J, Craddock L, Hong S, Liu ZM (2009) AMP-activated protein kinase suppresses LXR-dependent sterol regulatory element-binding protein-1c transcription in rat hepatoma McARH7777 cells. J Cell Biochem 106:414-426

40. Zelcer N, Tontonoz P (2006) Liver X receptors as integrators of metabolic and inflammatory signaling. J Clin Invest 116:607-614

41. Keech A, Simes RJ, Barter P et al (2005) Effects of long-term fenofibrate therapy on cardiovascular events in 9795 people with type 2 diabetes mellitus (the FIELD study): randomised controlled trial. Lancet 366:1849-1861

42. Nieuwdorp M, Stroes ES, Kastelein JJ (2007) Normalization of metabolic syndrome using fenofibrate, metformin or their combination. Diabetes Obes Metabol 9:869-878

43. Zambon A, Cusi K (2007) The role of fenofibrate in clinical practice. Diabetes Vasc Dis Res 4(Suppl 3):S15-S20

44. Danno H, Ishii KA, Nakagawa Y et al (2010) The liver-enriched transcription factor CREBH is nutritionally regulated and activated by fatty acids and PPARalpha. Biochem Biophys Res Commun 391:1222-1227

45. Han C, Wang J, Li L, Wang L, Zhang Z (2009) The role of LXR alpha in goose primary hepatocyte lipogenesis. Mol Cell Biochem 322:37-42

46. Pawar A, Botolin D, Mangelsdorf DJ, Jump DB (2003) The role of liver $\mathrm{X}$ receptor-alpha in the fatty acid regulation of hepatic gene expression. J Biol Chem 278:40736-40743 